\title{
Partikel- und chipbasierte Anreicherung von Bakterien für einen Point-of-Care-Nachweis
}

\author{
Karina Weber ${ }^{1,2,3}$, Susanne Pahlow ${ }^{1,3}$, Dana Cialla-May ${ }^{1,2,3}$, Jürgen Popp ${ }^{1,2,3}$ \\ ${ }^{1}$ Institut für Physikalische Chemie und Abbe Center of Photonics, FSU Jena, Helmholtzweg 4, \\ 07743 Jena, Deutschland \\ ${ }^{2}$ Leibniz-Institut für Photonische Technologien Jena, Mitglied im Leibniz-Forschungsverbund „Leibniz- \\ Gesundheitstechnologien“, Albert-Einstein-Straße 9, 07745 Jena, Deutschland \\ ${ }^{3}$ InfectoGnostics Forschungscampus Jena e.V., Philosophenweg 7, 07743 Jena, Deutschland \\ karina.weber@leibniz-ipht.de
}

\begin{abstract}
Zusammenfassung
Eine schnelle und sichere Identifikation von Krankheitserregern ermöglicht eine gezielte und schnelle Einleitung entsprechender Therapiemaßnahmen. Der erste Schritt zur erfolgreichen Identifikation von Bakterien ist die Anreicherung und Isolation aus komplexen Matrices wie Speichel, Sputum oder anderen respiratorischen Proben. Dies ist wichtig, um zu verhindern, dass Matrixbestandteile wie humane Zellen, Zellfragmente oder Proteine einen erfolgreichen Nachweis der Pathogene erschweren oder sogar verhindern. Im Rahmen des Beitrags präsentieren wir vielversprechende Strategien zur Probenvorbereitung die sowohl mit spektroskopischen als auch molekularbiologischen Detektionsverfahren kombiniert werden können. Zunächst diskutieren wir das Potential der partikelbasierten Anreicherung von Bakterien aus komplexen Proben. Hierbei werden Bakterien an die positivgeladene Partikeloberfläche adsorbiert und nach erfolgreicher Anreicherung mittels $\mathrm{pH}$-WertÄnderung desorbiert. Als Material zur Herstellung der Partikel können Polymere bzw. Glas eingesetzt werden. Darüber hinaus werden chipbasierte Ansätze präsentiert, bei denen Fängermoleküle wie Antikörper oder Siderophore auf Aluminiumoberflächen immobilisiert und die somit isolierten Bakterien mittels Raman-Spektroskopie identifiziert werden. Alle vorgestellten Verfahren haben das Potential in eine automatisierte Probenvorbereitung integriert zu werden um die gesamte Analysemesskette bis zum Point-of-Care-Nachweis abzudecken.
\end{abstract}

Keywords: Probenvorbereitung, Raman-Mikrospektroskopie, Amplifikation, PCR

\section{Einleitung}

Für den Nachweis von Mikroorganismen werden heutzutage immer noch häufig Ansätze verfolgt, welche eine Kultivierung der Erreger in geeigneten Nährmedien bedingen. Dieser Schritt führt dazu, dass diese Methoden sehr zeitaufwendig sind. In der medizinischen Diagnostik erweist sich diese Tatsache als besonders problematisch, da eine geeignete Therapie in den meisten Fällen nur dann initiiert werden kann, wenn der Erreger bekannt ist. Bei lebensbedrohlichen Erkrankungen wie einer Sepsis oder bei Patienten, deren Immunsystem stark beeinträchtigt ist, können derartige Verzögerungen des Therapiebeginns überaus kritisch sein. Dies illustriert die Notwendigkeit schnelle, kultivierungsunabhängige Nachweisverfahren für Mikroorganismen zu entwickeln.
Vielversprechende Ansätze liegen in der Anwendung verschiedener Amplifikationsmethoden, wie z.B. der PolymeraseKettenreaktion (polymerase chain reaction PCR), isothermale Amplifikationsverfahren (z.B. Helikase-abhängige Amplifikation (HDA)) oder der digitalen PCR. Neben diesem hochspezifischen, genotypischen Nachweis der Erreger, ist der Raman-spektroskopische Nachweis des Phänotyps eine schnelle und sensitive Alternative. Durch die Kombination eines Mikroskops mit einem RamanSpektrometer können Spektren einzelner Bakterienzellen innerhalb weniger Sekunden detektiert werden. Die Identifikation erfolgt anhand einer Datenbank, in der Spektren sämtlicher relevanter Spezies hinterlegt sind. Durch die spezifische chemische Information, welche durch die Raman-Spektren erhalten 


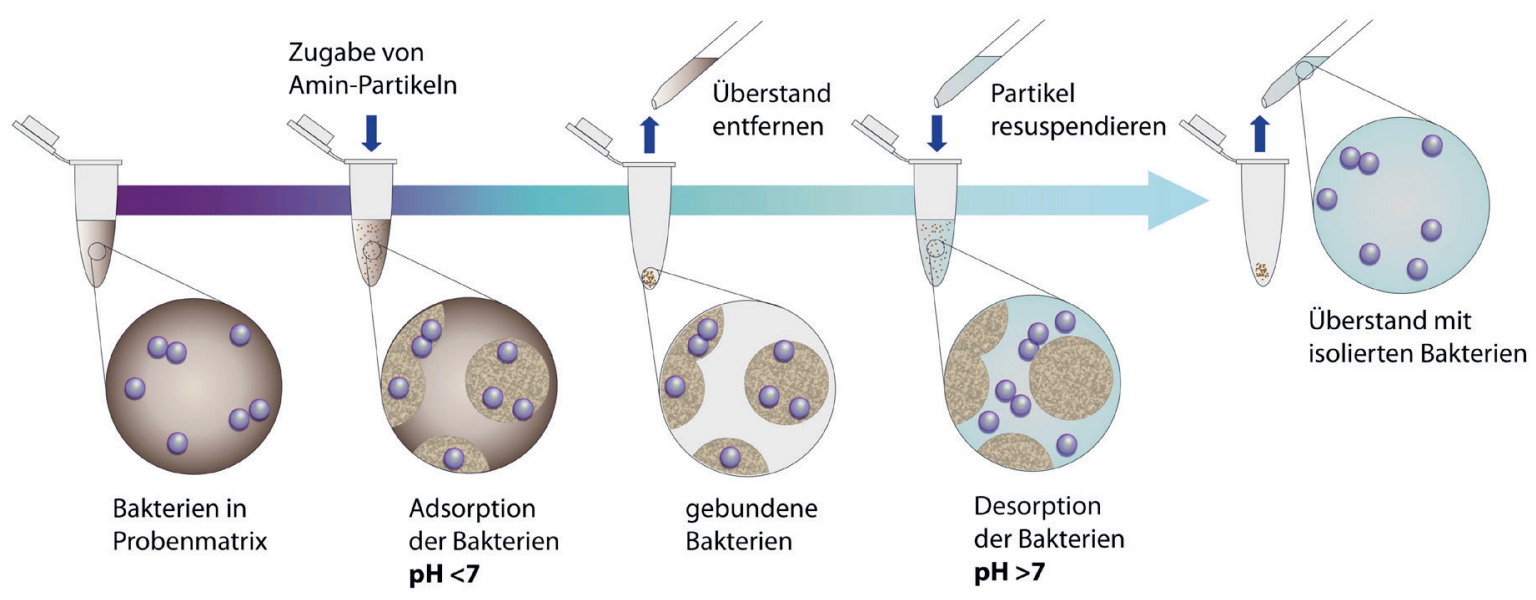

Abb. 1: Schematische Darstellung der Isolation von Bakterien mittels Amin-modifizierter Partikel.

wird, ist es möglich Bakterien eindeutig auf Spezies- oder sogar Stammebene nachzuweisen.

Bei dem für den Nachweis zu Verfügung stehenden Probenmaterial handelt es sich in der überwiegenden Mehrheit der Fälle um komplexe Matrices wie Körperflüssigkeiten, deren Bestandteile problematisch für die Anwendung der eben erwähnten Detektionsmethoden sind. Im Falle der PCR wirken Substanzen wie Harnstoff [1] oder Hämoglobin [2] inhibierend auf die Polymerase. Beim Raman-spektroskopischen Nachweis sind sämtliche Raman-aktive Probenbestandteile, wie zum Beispiel Proteine und Lipide hinderlich für eine eindeutige Identifizierung, da sich die entsprechenden Spektren mit denen der Bakterien überlagern. Aus diesem Grund ist die Entwicklung leistungsfähiger Probenvorbereitungsmethoden ein hoch relevantes Forschungsfeld. Im Folgenden werden wir exemplarisch erläutern wie partikel- und chipbasierte Ansätze mit PCR und Raman-Spektroskopie kombiniert werden können, um die vollständige Abdeckung der Prozesskette zu gewährleisten.

\section{Partikelbasierte Methoden}

Häufig besteht der Wunsch oder die Notwendigkeit verschiedene Erreger mit der angewendeten Isolationsmethode zu erfassen. Die Verwendung von Amin-modifizierten Oberflächen ist hier eine sehr vielversprechende Option. Über die Anwendung geeigneter Puffer kann die Protonierung der Amin-Gruppen gezielt gesteuert werden, so dass je nach Pufferbedingung unterschiedliche Ladungen an der Oberfläche der Partikel vorliegen. Da Bakterien im Allgemeinen eine negative
Oberflächenladung aufweisen, binden diese an die Partikel insofern dort eine positive Ladung vorliegt. [3] Dieser Ansatz ermöglicht ebenso eine gezielte Wiederablösung der Bakterienzellen, was gerade im Zusammenhang mit einer Ramanspektroskopischen Detektion attraktiv ist. Eine Untersuchung der Bakterien gebunden an Partikel ist in diesem Fall nicht möglich.

So konnte beispielsweise gezeigt werden, dass Polymerpartikel modifiziert mit Poly(ethylenimin) (PEI) sich sehr gut eignen um vitale Bakterien aus komplexen Bodenproben $\mathrm{zu}$ isolieren und sie anschließend Raman-spektroskopisch zu charakterisieren. [4] Anhand der RamanSpektren der untersuchten Spezies war es möglich diese mit einer hohen Genauigkeit zu identifizieren.

Die eben erwähnten PEI-Partikel ermöglichen ebenfalls eine Isolation von Nukleinsäuren aus Bodenproben. Für die DNA-Polymerase problematische Substanzen wie Huminsäuren und Tonmineralien lassen sich auf diesem Wege auf unkomplizierte Weise entfernen, so dass eine molekularbiologische Charakterisierung der Proben erfolgen kann.

\section{Chipbasierte Methoden}

Die Raman-Mikrospektroskopie ermöglicht es Bakterien auf Einzelzellebene zu untersuchen und $\mathrm{zu}$ identifizieren. Dieser Ansatz ist besonders dann attraktiv, wenn Proben mehr als eine Spezies erhalten, da jede Zelle separat behandelt wird. Eine Voraussetzung um qualitativ hochwertige Spektren zu erhalten ist dabei die Verwendung geeigneter Substrate, die selbst kein Raman-Signal liefern. Bewährt haben sich hier Metalloberflächen wie Nickel oder Aluminium. Bei der Entwicklung Raman-kompatibler 
Probenvorbereitungsstrategien sind chipbasierte Ansätze besonders interessant, da die Isolation und spektroskopische Untersuchung auf derselben Plattform durchgeführt werden können. Zuvor muss die Chipoberfläche mit spezifischen Fängermolekülen für Bakterien versehen werden, damit diese angereichert und von der umgebenden Probenmatrix separiert werden können. $\mathrm{Da}$ die Identifizierung der Bakterien anhand der typischen Raman-Spektren der verschiedenen Spezies erfolgt, ist die Verwendung speziesspezifischer Fängermoleküle nicht zielführend. Stattdessen sind Strategien sinnvoller, welche eine Anreicherung einer größeren Bandbreite an Erregern ermöglichen. Die Isolation verschiedener Sepsis-relevanter Bakterien mit anschließender Ramanspektroskopischer Untersuchung konnte mit Hilfe von Antikörpern realisiert werden, die gegen gemeinsame Zellwandbestandteile Gram-positiver (Lipoteichonsäuren) und Gramnegativer (Lipopolysaccharide) Bakterien gerichtet sind. [5]

Eine weitere Möglichkeit für eine breiter angelegte Isolation mehrerer Spezies besteht in der Ausnutzung eines Eisenaufnahmeweges von Bakterien, den sogenannten Siderophoren. Diese Verbindungen sind in der Lage dreiwertiges Eisen zu komplexieren und werden nach Bildung des entsprechenden Metallkomplexes über spezifische Aufnahmesysteme in die Zelle geschleust. Dieser Mechanismus lässt sich für eine chipbasierte Anreicherung heranziehen. Verschiedene Pseudomonaden, zum Beispiel $P$. aeruginosa und $P$. fluorescens, konnten mit einem Pyoverdin-Eisenkomplex auf einer Aluminiumoberfläche aus Trinkwasserproben angereichert und anschließend mittels RamanSpektroskopie erfolgreich identifiziert werden. [6]

\section{Schlussfolgerung}

Die Probenvorbereitung ist ein essentieller und für die erfolgreiche Anwendung verschiedener schneller und spezifischer molekularbiologischer und spektroskopischer Detektionsmethoden ein maßgeblicher Schritt in der Prozesskette. Die vorgestellten partikel- und chipbasierten Ansätze zeichnen sich durch eine breite Anwendbarkeit für verschiedene Spezies und Probenmatrices aus. Des Weiteren haben derartige Ansätze ein großes Potenzial für eine Automatisierung und zeichnen sich durch eine leichte Handhabbarkeit aus.

\section{Danksagung}

Wir danken dem Bundesministerium für Bildung und Forschung (BMBF) für die finanzielle Unterstützung der Projekte "EXASENS" (13N13856) und "InfectoGnostics" (13GW0096F).

\section{Literaturnachweis}

[1] G. Khan, H.O. Kangro, P.J. Coates, R.B. Heath, Journal of Clinical Pathology 44(5), 360-365 (1991); doi: 10.1136/jcp.44.5.360

[2] C. Schrader, A. Schielke, L. Ellerbroek, R. Johne, J Appl Microbiol 113, 1014-1026 (2012); doi: 10.1111/j.1365-2672.2012.05384.x

[3] A.T. Poortinga, R. Bos, W. Norde, H.J. Busscher, Surface Science Reports 47(1), 1-32 (2002); doi: 10.1016/S0167-5729(02)00032-8

[4] M. Schwarz, S. Kloß, S. Stöckel, S. Pollok, A. Holländer, D. Cialla-May, K. Weber, J. Popp, Anal. Bioanal. Chem. 409, 3779-3788 (2017); doi: $10.1007 / \mathrm{s} 00216-017-0320-z$

[5] S. Pahlow, S. Kloß, V. Blättel, K. Kirsch, U. Hüber, D. Cialla, P. Rösch, K. Weber, J. Popp ChemPhysChem 14, 3600-3605 (2013); doi: 10.1002/cphc. 201300543

[6] S. Pahlow, S. Stöckel, S. Pollok, D. Cialla-May, P. Rösch, K. Weber, J. Popp, Anal. Chem. 88(3), 1570-1577 (2015); doi: 10.1021/acs.analchem.5b02829 